\title{
Deutsch-Mexikanische Juristenvereinigung e.V.
}

\author{
Von Gerhard Scheffler
}

Am 30. Juni 1988 ist in München die Deutsch-Mexikanische Juristenvereinigung (Effnerstraße 38, 8 München 81) gegründet worden, die es sich zum Ziel setzt, die wechselseitige Kenntnis und das Verständnis der beiden Rechtsordnungen zu fördern, indem u.a. Informationen ausgetauscht, zweisprachige Publikationen veröffentlicht und Vorträge gehalten werden. Die Verwirklichung der Vereinsziele soll laut Satzung in Abstimmung mit der Deutsch-Mexikanischen Gesellschaft e.V. erfolgen, die demgemäß auch eine finanzielle Starthilfe gewährte. In den ersten Vorstand wurden gewählt Dr. Karl August Prinz von Sachsen Gessaphe, Präsident, Professor Dr. Peter Winkler von Mohrenfels, 1. Vizepräsident, und Prof. Dr. Volkmar Gessner, 2. Vizepräsident. Ein Generalsekretär und ein Schatzmeister wurden bestellt. Zwei Beisitzerpositionen blieben offen, insbesondere zwecks Besetzung durch mexikanische Juristen. Die Vereinigung ist inzwischen als Verein eingetragen.

Im (jährlichen) Mitteilungsblatt El Mensa jero, Nr. 1 vom Mai 1989 sind zum einen der Festvortrag Volkmar Gessner: Traditionelle und modeme Zivilgerichtsbarkeit in Mexiko, sowie zwei Beiträge zum IPR (Referate der Vortragsveranstaltung vom 9. Februar 1989) aus deutscher und mexikanischer Sicht abgedruckt. In der Zeit vom 8.-10. Juni 1989 fand im Beisein mexikanischer Konsulatsbeamter der 1. Jahreskongre $\beta$ der Vereinigung im Dominikanerkloster St. Albert in 5303 Bornheim statt. Die nächsten Jahrestagungen sind für Juni 1990 in München für 1991 in Mexico-Stadt vorgesehen. Die Gründung einer Schwestervereinigung in Mexiko steht indessen noch aus.

Die Vereinigung vermittelt Referendare für eine Wahlstation nach Mexiko sowie Kontakte zu Anwälten für die Lösung deutsch-mexikanischer Rechtsprobleme. Der jährliche Mitgliedsbeitrag beträgt derzeit DM 60,-; Referendare DM 30,-, Studenten DM 15,-. 